\title{
Mutual replacement reactions in alkali feldspars II: trace element partitioning and geothermometry
}

Ian Parsons - Charles W. Magee - Charlotte M. Allen •

J. M. G. Shelley · Martin R. Lee

Published online: 3 February 2009

(C) Springer-Verlag 2009

\section{Erratum to: Contrib Mineral Petrol}

\section{DOI 10.1007/s00410-008-0358-1}

The original version of this article unfortunately contained a mistake. It affects the bottom line of numbers in Table 3.
Both the numbers 382 and 392 should be 340 . In addition, two unwanted lines of text were printed below the table. The corrected table is given here.

The online version of the original article can be found under doi:10.1007/s00410-008-0358-1.

I. Parsons ( $\square)$

Grant Institute of Earth Science, University of Edinburgh,

West Mains Road, Edinburgh EH9 3JW, UK

e-mail: ian.parsons@ed.ac.uk

C. W. Magee - C. M. Allen · J. M. G. Shelley

Research School of Earth Sciences, Australian National

University, Canberra, ACT 0200, Australia

M. R. Lee

Department of Geographical and Earth Sciences,

University of Glasgow, Lilybank Gardens,

Glasgow G12 8QQ, UK 
Table 3 Temperature estimates for exsolution and coarsening events

\begin{tabular}{|c|c|c|c|c|c|c|}
\hline Crystal & & & 140177 & KB22 & KS55B & KS55C \\
\hline Bulk An of braid, mol\% & & & 3.85 & 1.91 & 1.82 & 0.93 \\
\hline Event & Basis & $T$ from & $T^{\circ} \mathrm{C}$ & $T^{\circ} \mathrm{C}$ & $T^{\circ} \mathrm{C}$ & $T^{\circ} \mathrm{C}$ \\
\hline (1) Minimum $T$ for crystal growth & $\begin{array}{l}\text { Ternary solvus isotherm at } 0.1 \mathrm{GPa} \text {, } \\
\text { Elkins and Grove (1990) }\end{array}$ & & 830 & 770 & 750 & 740 \\
\hline \multicolumn{7}{|l|}{ Braid perthite formation } \\
\hline (2) Spinodal decomposition & $\begin{array}{l}\text { (1) minus } T \text { interval between coherent } \\
\text { and strain-free solvi, Brown and } \\
\text { Parsons (1984, Fig. 9) }\end{array}$ & & 770 & 710 & 690 & 680 \\
\hline $\begin{array}{l}\text { (3) Coarsening, ordering, } \\
\text { twinning and interface } \\
\text { rotation }\end{array}$ & $\begin{array}{l}\text { Brown and Parsons (1984; Fig. 8) } \\
\text { Brown and Parsons (1989, Fig. 3) }\end{array}$ & & \multicolumn{4}{|c|}{$\begin{array}{l}\text { Continuous into low microcline } \\
\text { field at } 450-420^{\circ} \mathrm{C}\end{array}$} \\
\hline \multicolumn{7}{|l|}{ Patch perthite formation } \\
\hline \multirow{4}{*}{$\begin{array}{l}\text { (4) Strain-free ternary } \\
\text { feldspar pairs, disordered }\end{array}$} & \multirow{4}{*}{$\begin{array}{l}\text { Two-feldspar tie-lines defined by } \\
\text { average analyses in Table } 1 \text {, at } \\
0.1 \mathrm{GPa} \text {, Elkins and Grove (1990) }\end{array}$} & Average & 504 & $487^{\mathrm{a}}$ & 502 & 506 \\
\hline & & $T_{\mathrm{Ab}}$ & 452 & 489 & 379 & 376 \\
\hline & & $T_{\mathrm{An}}$ & 504 & 489 & 502 & 506 \\
\hline & & $T_{\text {Or }}$ & 504 & 482 & 502 & 506 \\
\hline $\begin{array}{l}\text { (5) Correction of (4) for } \\
\mathrm{Si}-\mathrm{Al} \text { ordering }\end{array}$ & Brown and Parsons (1984, Fig. 9) & $\begin{array}{l}\text { Concordant } \\
T \text { only }\end{array}$ & 584 & 567 & 582 & 586 \\
\hline \multicolumn{7}{|l|}{ Third stage exsolution } \\
\hline $\begin{array}{l}\text { (6) Maximum } T \text { for coherent } \\
\text { nucleation of albite lamellae }\end{array}$ & $\begin{array}{l}\text { Ordered coherent solvus of Yund (1974). } \\
\text { Based on Or only, An ignored }\end{array}$ & & 424 & 461 & 340 & 340 \\
\hline (7) Formation of misfit dislocations & & \multicolumn{5}{|c|}{$\begin{array}{l}\text { Nucleation below (6), following coarsening. } \\
\text { Variable with bulk composition of Or-rich patch }\end{array}$} \\
\hline
\end{tabular}

a Temperatures in bold are concordant, as defined by Fuhrman and Lindsley (1988) 